\title{
Multidimensional Fatigue Inventory
}

National Cancer Institute

\section{Source}

National Cancer Institute. Multidimensional Fatigue Inventory. NCI Thesaurus. Code C54719.

A 20-item self-report instrument designed to measure fatigue. It covers the following dimensions: General Fatigue, Physical Fatigue, Mental Fatigue, Reduced Motivation and Reduced Activity. 